\title{
Research on the Equipment Fault Diagnosis Based on GN-BP Neural Network
}

\author{
Jian $\mathrm{Du}^{1,2, a}$, Yu-cai Dong ${ }^{3, \mathrm{~b}}$, Jing Xia ${ }^{4, c}$, Huizhen $\mathrm{Li}^{5, \mathrm{~d}}$ \\ 1,3,4,5 Department of Fundamental, Academy of Armored Forced Engineering, Beijing, 100072, \\ China \\ ${ }^{2}$ Department of Mathematics, Texas A\&M University, College Station, 77840, USA \\ aemail: \\ jiandu2531@163.com, bemail:ycdong@163.com, cemail:jxia@163.com, demail:hzli@163.com
}

Keywords: Fault diagnosis; GA-BP; Network training; Data analyses

\begin{abstract}
Neural network provides a new research method for equipment fault diagnosis with its inherent memory ability, self-learning ability and strong fault tolerance. In the basis of equipment fault diagnosis characterized mathematical, the research established the model of fault diagnosis for equipment based on GA-BP, and the diagnosis model has been applied in the theoretical analyses and test for fault diagnosis of gear box. Experimental results show that equipment fault diagnosis technology based on GA-BP neural network, it can optimize the tactics with genetic algorithm when the network trained model, and adjust precision using BP network, the model can accurately diagnosis the types of fault for equipment. The model of GA-BP neural network has higher prediction accuracy and adaptability than the traditional BP neural network and has important application in the field of fault diagnosis.
\end{abstract}

\section{Introduction}

Traditional analysis and design method of equipment fault diagnosis is established on the accurate mathematical model. In actually, the system has the complexity, nonlinear, time-varying and uncertainty, and it is difficult to establish the accurate mathematical model and to guarantee the accuracy of diagnosis. The neural network provides a new theoretical methods and technical technology for equipment fault diagnosis.[1]

Each the nodes and the weight of Back Propagation Neural Network(BPNN) affect the output, its adaptation and global approximation process is time-consuming, so network convergence speed is slow. At the same time, BPNN is non-convex problem of the gradient descent, easy to fall into local minimum value and lead to wrong working mode. Genetic Algorithm(GA) is a kind of robust adaptive optimization method based on biological evolution principle. On the basis of the samples fitness function, GA guide the study by select, crossover and mutation operation for the initial population and determine the search direction.[2] Due to search the soultion by the way of population, it can seek the optimal solution by random method in the multiple area of the global solution space. Considering the disadvantage of BPNN and the advantage of the GA, GA-BP is a technical strategy of improving the equipment fault diagnosis accuracy.

\section{The mathematical description of the equipment fault diagnosis}

If all the possible state of diagnosed equipment combinate into state space $X$, and all observable value range of the equipment constitute feature space $Y$. Assuming that the equipment is in the state space $X 1$, then the observable value of equipment is considerable character value $Y 1$, namely there is a mapping relationship $K$ :

$$
K: X 1 \rightarrow Y 1
$$

On the contrary, a certain characteristics $Y 1$ also correspond in a certain state $X 1$ in a state space, namely there is a mapping relationship $G$ : 
$G: Y 1 \rightarrow X 1$

If the feature space and the state space is to meet single surjective of one-to-one relationship, it can only determine the equipment state according to the observable values, and vice versa. in equipment fault diagnosis, the purpose is determine what kind of state in the state space for equipment by measurable characteristic vectora, and it is to find the map $G$.[3]

\section{The training and prediction principle of BPNN}

\section{The basic thoughts of network training.}

BPNN is a kind of multilayer feedforward network according to the error back Propagation algorithm training. Its learning rule is to use the steepest descent method, by back propagation to constantly adjust the network weights and threshold, to minimize the squares sum of error for the network. thus to complet the process of information extraction and memory. The topology structures of three layer BPNN model include input layer, the hide layer and output layer, the topology structure as shown in figure 1.[4]

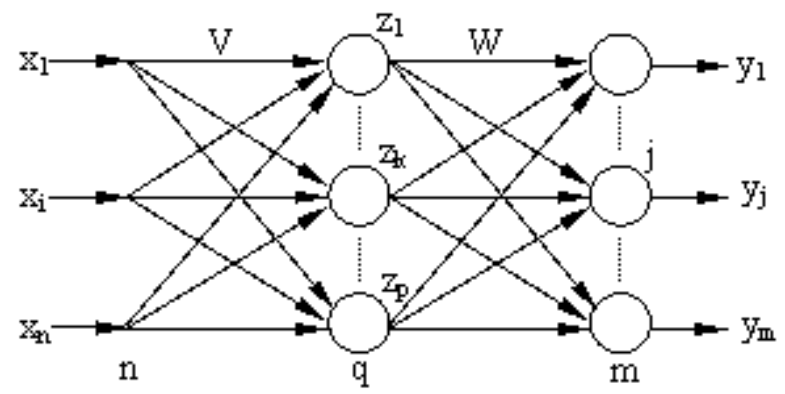

Fig.1. The topology structures of three layer BPNN model

The input layer of BPNN has $n$ nodes, the hide layer has $q$ nodes, the output layer has $m$ nodes,the weights between input layer and hide layer is $v_{k i}$, the weight between hide layer and output layer is. The transfer function of hide layer is $f_{1}(\cdot)$, the transfer function of output layer is $f_{2}(\cdot)$, then the output of the hide layer node is shown as (to write threshold into the sum item)

$$
z_{k}=f_{1}\left(\sum_{i=1}^{n} v_{k i} x_{i}\right)(k=1,2, \cdots, q)
$$

The output of the output layer node is shown as

$$
y_{j}=f_{2}\left(\sum_{k=0}^{q} w_{j k} z_{k}\right) \quad(j=1,2, \cdots, m)
$$

At this time, the BPNN has completed the approximation mapping for $n$ dimensional space vector for $m$ dimensional space.

\section{The basic idea of Network to predict.}

The purpose of network training is the nonlinear learning for a variety of modes by using each neurons and structure corresponding weights and threshold, and a number of learning information is stored in various weights and thresholds. As long as the training sample is large enough, the information of the network storage is more fully, the ability of distinguish patterns is stronger.[5] In fact, the equipment fault diagnosis based on neural network learn and memory the cause mode of the fault failure in the process of training past samples, thus it can make correct diagnosis to existing fault. 


\section{The equipment fault diagnosis model based on GN-BPNN}

\section{Basic idea of GN-BPNN.}

The research idea on equipment fault diagnosis based on GA-BPNN is to solve knowledge expression of the diagnostic equipment, knowledge acquisition and reasoning mechanism by using the neural network adaptive learning, distributed storage, associative memory and massively parallel processing. Its main technology is to optimize the initial weights and threshold of neural network by GA as it given initial neural network. When the algorithm reaches a certain convergence requirements, then GN-BP use neural network to carry on the second training for improving the training accuracy and speed and to avoid local optimum.

The model structure design of GA - BPNN.

\section{1)The model parameter selection of GA - BPNN}

In order to improve the range of the input vector, and make the range of output vector are within the scope $(0,1)$, select the function tan sig as the activation function of hidden layer neurons, select the function $\log$ sig function as the activation function of output layer neurons. [6]

Function of the $\tan \operatorname{sig}$ is shown as follows:

$$
f(x)=\frac{1-\exp (-x)}{1+\exp (-x)}
$$

Function the logsig is shown as follows:

$$
f(x)=\frac{1}{1+\exp (-x)}
$$

To take 0.05 as learning rate. In this paper, using genetic algorithm to generate the initial weights, and try on take data of normalized experiment for simulation.

\section{2)The code of genetic algorithm}

In this paper, using real number to code. In the process of encoding, to take each weight and threshold of neural network as the gene of GA, to generate randomly a set of distribution of weights and threshold in $[-1,1]$ for neural network, and to connect the weights and thresholds in order into a long list.[7]

\section{3)Population initialization}

Because the BP algorithm itself has optimization ability, it doesn't need choice large population, the population size can be set between 10 to 30. After repeated experiments and debugging, the size of the population of genetic algorithm can be set as 20 .

\section{4)The fitness function}

Mean square error of the neural network output is as follows:

$$
E=\frac{1}{N} \sum_{s} \sum_{m}\left(t_{m}-o_{m}\right)^{2}
$$

In formula, $s$ denote the number of samples, $m$ denote the number of output layer node, $t_{m}$ denote the desired output of neural network, $o_{m}$ denote the input of the neural network.[8]

The fitness function can be defined as:

$$
f=\frac{1}{E+1}
$$

Type the denominator contains constant 1 goal is to prevent in E very small cases, spread function.

5)To determine the genetic operators

The selection operator . The selection probability is as follows:

$$
P_{i}=\frac{f_{i}}{\sum_{i=1}^{m} f_{i}}
$$

Among them, $1 \leq i \leq m, m$ denote the number of individual in the group, $f_{i}$ is the fitness of 
selected individuals.

The crossover operator. The crossover rate is calculated by the formula.

$P_{c}= \begin{cases}P_{c 1} & f^{\prime} \geq f_{a v g} \\ P_{c 2} & f^{\prime}<f_{\text {avg }}\end{cases}$

In formula, $f^{\prime}$ is the fitness value of two individual for crossover operation, $f_{a v g}$ is the average fitness of the individual for the population. $P_{c 1}, P_{c 2}$ value can be adjusted according to the final search results for the genetic algorithm. In this paper, $P_{c 1}$ and $P_{c 2}$ is set respectively to 0.5 and 0.9 .

The mutation operator .The mutation rate

$P_{m}= \begin{cases}P_{m 1} & f \geq f_{\text {avg }} \\ P_{m 2} & f<f_{\text {avg }}\end{cases}$

In formula, $f$ is the fitness value of two individual for crossover operation, $f_{\text {avg }}$ is the average fitness of the individual for the population. $P_{m 1}, P_{m 2}$ value can be adjusted according to the final search results for the genetic algorithm. In this paper, $P_{m 1}$ is set to 0.001 , and $P_{m 2}$ is set to 0.01 .

6)The optimization process of GA-BP network

The key of GA-BP modeling is to determine the structure layers of the BPNN, the number of neurons for each layer, gene chain formed by the weights and threshold, the population size is $\mathrm{N}$, two constant $k_{1}, k_{2}$ of crossover probability $p_{c}$ and iteration precision $s_{1}, s_{2}$. When the iteration meet established termination conditions, it will decode the individual values of the optimal fitness, and provide the initial weights and threshold of BP network training again for the next step (they respectively are $W 、 V 、 \theta$ and $T$ ).

In specific training, the samples is the matrix $S_{u \times n}$, the goal is matrix $G_{u \times p}$. Obviously, the node number of input layer is $n$, the node number of output layer is $p$, the node number of hidden layer are determined by empirical formula $m=\sqrt{n p}$. The code length of individual (chromosomes) is

$$
L=n m+m p+m+p
$$

\section{The application of GA-BPNN model in the equipment fault diagnosis}

Gear box is an indispensable general parts of transfer movement and power for equipment mechanical. If gearbox occurs fault, it will not only result in its own damage, cannot continue to run, also lead to the entire power equipment stopping run. Therefore, it is very important significance for the safety of equipment which the equipment fault diagnosis of gearbox.

\section{Information extraction for equipment fault diagnosis.}

The study collected the sample data of a certain state for gear box. The data are shown in table 1 and 2.

Table.1. The training data of gearbox state

\begin{tabular}{|c|c|c|}
\hline Data serial number & Training data & Gear state \\
\hline 1 & 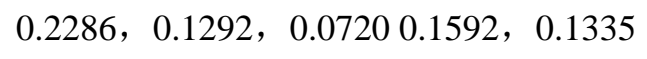 & Trouble - free \\
\hline 2 & $0.2090, \quad 0.0947,0.13930 .1387,0.2558$ & Trouble - free \\
\hline 3 & $0.2603,0.1715,0.07020 .2711,0.1491$ & Tooth root fracture \\
\hline 4 & 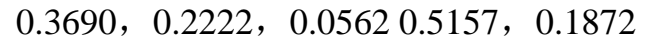 & Tooth root fracture \\
\hline 5 & $0.0724,0.1909,0.13400 .2409, \quad 0.2842$ & Snaggletooth \\
\hline 6 & $0.2634, \quad 0.2258, \quad 0.11650 .1151, \quad 0.1074$ & Snaggletooth \\
\hline
\end{tabular}


Table.2. The test data of a gearbox state

\begin{tabular}{|c|c|}
\hline Data serial number & Test data \\
\hline 7 & $0.2599,0.2235,0.1201,0.1171,0.1102$ \\
\hline 8 & $0.2593,0.1800,0.0711,0.2801,0.1501$ \\
\hline
\end{tabular}

\section{The data analysis and processing.}

The obvious fault in frequency domain is on the edge of meshing frequency, so the research choose the several characteristics of the frequency domain. The data in Table 1 and table2 have different units and magnitude, the research normalize the data, set three kinds of gear fault modes for gear: trouble-free: $(0,1)$; root fracture $(0,0)$; broken teeth $(0,1)$.

To analysize simulation results for GA - BP neural network model.

By using the sample data in table1, the research train net with training function of different algorithms, and it make different input gets different output. When the error can satisfy the demands to stop training, the weight coefficient of network is indicators of internal representation for fault diagnose.

In order to get better training simulation results, the research simulate the fault diagnosis of equipment based on BPNN. The testing process are shown in figure 2.

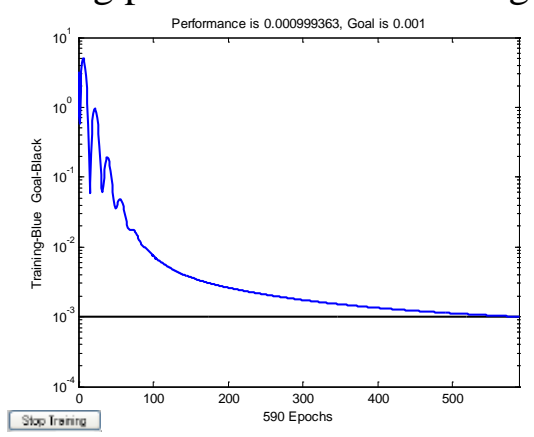

Fig.2. The process of network training

The research train neural network by using the data in table 1 . It take the test data into to the trained network and attain the training goals after the training time met 590 generation. Getting the output of the test data 7 is $0.0000,1.0000,0.0014$, the output of the test data 8 is $0.0000,0.0001$, 0.9999. To contrast the previously defined failure mode, the test data 7 correspond tooth root fracture and the test data 8 correspond broken teeth.

\section{Conclusion}

Through data analysis and example simulation, it prove effective that the model of fault diagnosis for equipment based on GA-BPNN. The model not only improve the technology of fault diagnosis and maintenance, but also classify the fault type happened in the past and find the relationship among fault, judge the future trend of the work status for various parts of the equipment according to the test data in status. The research has a great significance for preventing equipment accidents and reducing the maintenance cost.

\section{References}

[1]Wang X, Liu C W, Bi F R et al. Fault diagnosis of diesel engine based on adaptive wavelet packets and EEMD-fractal dimension[J].Mechanical Systems and Signal Processing, 2013, 41(1/2): 581-597.

[2]Wang Y F, Xue C, Jia X H et al. Fault diagnosis of recipro-cating compressor valve with the method integrating acoustic emission signal and simulated valve motion [J]. Mechanical Systems and Signal Processing, 2015, 56-57: 197-212.

[3]Liu X F, Bo L, Luo H L. Bearing faults diagnostics based on hybrid LS-SVM and EMD method [J]. Measurement, 2015, 59: 145-166. 
[4]Jegadeeshwaran R, Sugumaran V. Fault diagnosis of auto-mobile hydraulic brake system using statistical features and support vector machines [J]. Mechanical Systems and Signal Processing, 2015, 52-53: 436-446.

[5]Bin G, Gao J, Li X Early fault diagnosis of rotating ma-chinery based on wavelet packets-empirical mode decomposition feature extraction and neural network. Mech Syst Signal Process, 2012, 27:696-711

[6]Samanta B, Al-Balushi K R, Al-Araimi S A. Artificial neu-ral networks and support vector machines with genetic al-gorithm for bearing fault detection [J]. Engineering Appli-cations of Artificial Intelligence, 2003, 16(7/8): 657-665.

[7]Liu H, Tian H, Chen C, Li Y An experimental investi-gation of two wavelet-MLP hybrid frameworks for wind speed prediction using GA and PSO optimization. Int J Electr PowerEnergy Syst, 2013 52(1):161-173

[8]Liu H, Tian $\mathrm{H}$, Chen $\mathrm{C}$, Li Y An experimental investi-gation of two wavelet-MLP hybrid frameworks for wind speed prediction using GA and PSO optimization. Int J Electr Power Energy Syst, 2013, 52(1):161-173 